\title{
Polarized ion beams in atomic physics research
}

\author{
A. Surzhykov ${ }^{* \dagger, 1,2}$, A. N. Artemyev ${ }^{1,2}$, V. A. Yerokhin ${ }^{1,2,3}$, S. Fritzsche ${ }^{2,4}$, and \\ Th. Stöhlker ${ }^{1,2,5}$ \\ ${ }^{1}$ Physikalisches Institut, Universität Heidelberg, D-69120 Heidelberg, Germany \\ ${ }^{2}$ GSI Helmholtzzentrum für Schwerionenforschung GmbH, D-64291 Darmstadt, Germany \\ ${ }^{3}$ St. Petersburg State Polytechnical University, St. Petersburg 195251, Russia \\ ${ }^{4}$ Department of Physics, University of Oulu, P.O. Box 3000, FI-90014 Finland \\ ${ }^{5}$ Helmholtz-Institut Jena, D-07743 Jena, Germany \\ ${ }^{\dagger} E$-mail: surzephysi.uni-heidelberg.de
}

\begin{abstract}
A review is presented of recent theoretical progress in atomic physics studies with spin-polarized heavy ions. Special emphasis is placed on the diagnostics of ion beams at storage rings. In particular, we show how the radiative electron capture from low $-Z$ target atoms may serve as a very sensitive probe of heavy-ion spin polarization. The role of the electron-electron interactions in such a polarization-measurement method is discussed in detail.
\end{abstract}

8th International Conference on Nuclear Physics at Storage Rings-Stori11,

October 9-14, 2011

INFN, Laboratori Nazionali di Frascati, Italy

\footnotetext{
*Speaker.
} 


\section{Introduction}

During the last decade, studies with beams of spin-polarized, heavy ions at storage rings have attracted much attention in many areas of modern research. In atomic physics, for example, a number of spin-dependent collision experiments have been proposed to better understand the relativistic, many-body and QED effects on the structure and dynamics of heavy atomic systems. Moreover, application of polarized heavy ion beams opens up a very promising way to explore atomic parity non-conservation (PNC) phenomena as well as to search for electric dipole moments of heavy nuclei; a proposed test of the Standard Model of particles and interactions [, \, []]. Along this line, a series of experiments are planned to be carried out at the Facility for Antiproton and Ion Research (FAIR) that is now under construction in Darmstadt. Any practical realization of the spin-dependent studies will however require efficient methods for the production and operational control of polarized ion beams. In this contribution we shall therefore outline recent theoretical proposals for dealing with these two key problems. Special attention will be paid to the diagnostics of the ion spin polarization. In particular, we will recall the recent proposal to employ radiative electron capture (REC) as a probe process, whose properties are sensitive to the spin states of heavy ions [䧃]. It will be shown that the implementation of this scheme requires an accurate account of the $e-e$ interaction effects as occur both in a target atom and in a few-electron projectile ion.

\section{Production of polarized heavy ions}

Efficient schemes for the production of spin-polarized ion beams at storage rings are currently under intense discussion. In the work by Prozorov and co-workers [15, 目] for example, an optical pumping of the Zeeman sublevels of the hyperfine states of hydrogen-like species has been proposed in order to achieve the predominant population of some particular substate $\left|F M_{F}\right\rangle$. Since such a state results from the coupling of the electron and the nuclear momenta, $\mathbf{F}=\mathbf{j}+\mathbf{I}$, it requires that the nuclear spin be polarized as well. The key parameter in such a scenario is the so-called degree of ion polarization [可:

$$
\lambda_{F}=\sum_{M_{F}} n_{M_{F}} M_{F} / F
$$

where $n_{M_{F}}$ is the relative population of the hyperfine sublevel $\left|F M_{F}\right\rangle$. The realization of such a "laser-excitation" polarization scheme obviously requires an effective tool for the accurate measurement of the degree $\lambda_{F}$. One has to find, therefore, a physical process whose properties are sensitive enough to the spin state of the high- $Z$ hydrogen-like projectiles and that can be measured sufficiently simply. In the next Section, we discuss just such "probe" processes and especially focus on the radiative electron capture.

\section{Diagnostics of ion polarization}

A number of theoretical studies were reported recently on the spin-diagnostics of heavy, highly-charged ions at storage rings. For example, Bondarevskaya and co-workers [ [ $]$ ] have proposed using the dependence of the hyperfine quenching (HFQ) on the polarization of the ion placed in an external magnetic field. The information on the population of the hyperfine substates $\left|F M_{F}\right\rangle$ 
can be obtained also from the analysis of the linear polarization of $\mathrm{x}$-rays emitted in hyperfinequenched characteristic transitions [ [8]. Yet another alternative and very promising scheme employs the radiative electron capture (REC); the process which is dominant in relativistic collisions of high $-Z$ projectiles with low $-Z$ target atoms. Below we will show how the polarization properties of the REC photons are related to the degree of ion polarization $\lambda_{F}$ and, hence, provide an efficient tool for the beam diagnostics.

\subsection{Polarization transfer in the radiative electron capture}

The last few years have witnessed significant progress in the development of solid-state x-ray

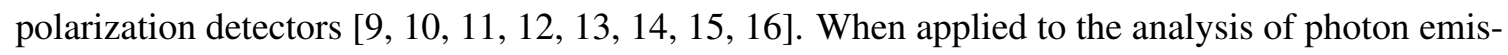
sion from heavy highly-charged ions, these detectors grant more insight into the atomic structure and dynamics in the high- $Z$ domain. Relativistic, quantum electrodynamic (QED), and even parityviolation (PV) phenomena, which are usually difficult to isolate when probing total cross sections, often become visible in polarization-sensitive studies. Recently, a number of experiments have been initiated to analyze the linear polarization of light emitted in the radiative electron capture of loosely bound target electrons by high $-\mathrm{Z}$ ions. This process nowadays attracts much attention since it plays an important role for the $\mathrm{x}$-ray spectroscopy of heavy atomic species and provides a unique tool for exploring the fundamental process of light-matter interaction in the realm of strong electromagnetic fields and high energies [ㅍ]].

Apart from the fundamental interest in REC polarization studies, their application towards the diagnostics of spin-polarized heavy-ion beams currently attracts much attention. For instance, we have recently argued that REC can be employed as a "probe" process, whose properties are extremely sensitive to the spin states of the collision system [ [4, [8]]. It was shown, in particular, that the tilt angle $\chi_{0}$ of the linear polarization of the recombination photons, defined with respect to the reaction plane (spanned by the directions of the incident beam and emitted light), is uniquely defined by the ion degree of polarization $\lambda_{F}$ :

$$
\tan 2 \chi_{0}=\lambda_{F} \frac{I-1 / 2}{I+1 / 2} \mathscr{R}\left(Z, T_{p}\right),
$$

where $I$ is the nuclear spin and $\mathscr{R}$ is some function which depends only on collision parameters such as the projectile velocity and charge. As seen from this expression, the capture of electrons by unpolarized hydrogen-like ions, $\lambda_{F}=0$, always leads to an emission of light which is polarized either within or perpendicular to the reaction plane $\left(\chi_{0}=0^{\circ}\right.$ or $\left.90^{\circ}\right)$, while any contribution from a nonzero $\lambda_{F}$ parameter will rotate the polarization ellipse out of the reaction plane. The measurement of the tilt angle $\chi_{0}$, therefore, provides direct access to the degree $\lambda_{F}$ of the ion polarization without needing that the Stokes parameters or the shape of polarization ellipse be analyzed in detail.

Figure $\square$ displays the tilt angle $\chi_{0}$ as calculated, for example, for $K$-REC of completely polarized $\left(\lambda_{F}=1\right)$ hydrogen-like europium ions with nuclear spin $I=5 / 2$ and energies in the range 100 $\leq T_{p} \leq 600 \mathrm{MeV} / \mathrm{u}$. As seen from this figure, the effect of the ion polarization becomes particularly remarkable for the forward emission of the recombination photons. Note however that $\chi_{0}$ is not defined at the emission angle $\theta=0^{\circ}$ ( or $\theta=180^{\circ}$ ), because photon emission in either the forward or backward direction does not break the axial symmetry for the collision system. For the angle $\theta$ 

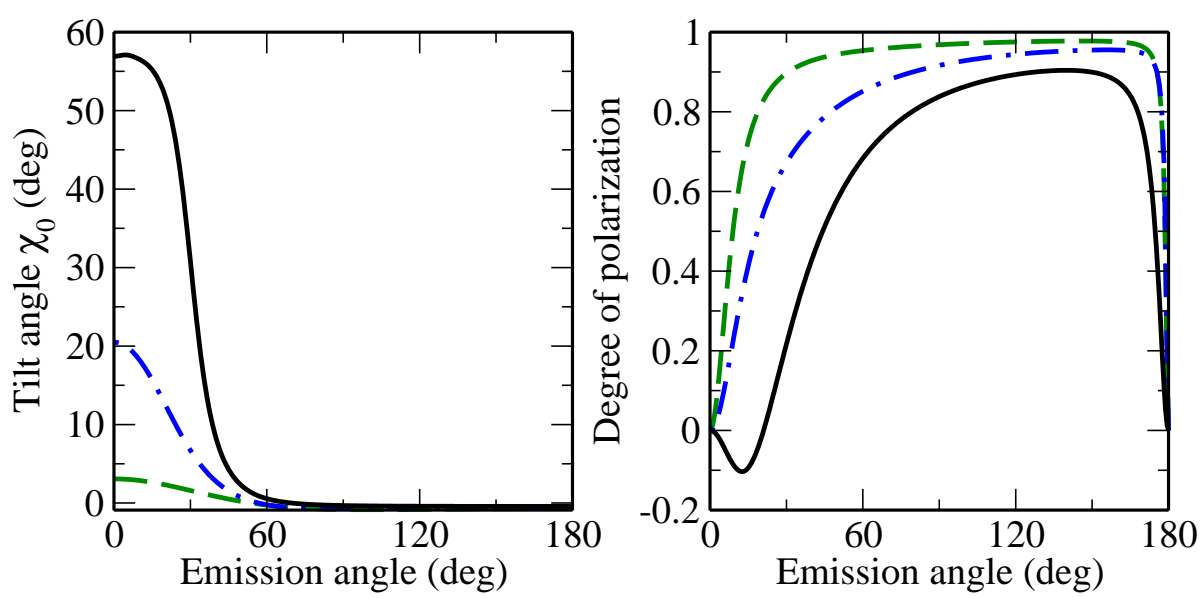

Figure 1: The tilt angle $\chi_{0}$ (left panel) and the degree (right panel) of the linear polarization of the photons which are emitted in the radiative recombination of an electron into the ground state of (initially) polarized hydrogen-like europium $\mathrm{Eu}^{62+}$ ion with projectile energies of 100 (dashed line), 300 (dash-and-dotted line), and $600 \mathrm{MeV} / \mathrm{u}$ (solid line). Results compiled from Ref. [प]]

$=0^{\circ}$, therefore, the linear polarization of the light must always be zero (see right panel of Fig. $\mathbb{W}$ ). At larger angles of, say $10^{\circ}<\theta<60^{\circ}$, however, the (degree) of linear polarization becomes large enough for experiments and preferable for first investigations on the polarization of ion beams.

\subsection{Target effects on the polarization transfer}

The theoretical analysis of the REC polarization transfer, outlined in the previous section, is based on the independent particle model for the description of the final (helium-like) ionic states as well as on the assumption that the incident electrons are free. For accurate diagnostics of spinpolarized ion beams it is therefore important to question how the REC polarization is affected by inter-electronic interactions, as occur both in (i) a target atom and (ii) in an ultimately few-electron projectile ion. In order to understand the role of "target effects", we have recently employed the impulse approximation, appropriate for fast collisions of heavy projectiles with low- and medium$Z$ targets. Within this approximation, the projectile ion and target atom are treated in an asymmetric fashion. Hence, while the motion of the electron in the initial-continuum and final-bound states is considered as driven solely by the field of the projectile nucleus, the target charge just provides the electron momentum distribution in the initial channel [20]:

$$
\Psi_{i}(\mathbf{r}, t)=\frac{\mathrm{e}^{-i \varepsilon_{p} t / \gamma}}{\gamma} \int \mathrm{d}^{3} \mathbf{p} \phi_{i}\left(\mathbf{p}_{\perp} ; \kappa_{z}\right) \sqrt{\varepsilon_{p}} \psi_{\mathbf{p} m_{s}}(\mathbf{r}) \mathrm{e}^{-i \mathbf{p} \cdot \mathbf{R}(t)} .
$$

Here, $\mathbf{R}(t)$ is the position of the nucleus of the (target) atom as seen by the projectile, $\mathbf{p}$ and $\varepsilon_{p}=$ $\sqrt{c^{2} p^{2}+m^{2} c^{4}}$ describes the asymptotic momentum and energy of the (originally free) electron and a convolution is made over the momenta of the bound target electron. The further steps in the evaluation of the transition amplitudes and polarization parameters then follow very similar lines as in standard perturbation theory, while the impact parameter dependence here remains explicitly due to the decomposition of the $\mathbf{R}(t)$ into parallel and perpendicular components. 


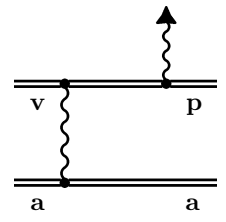

(1)

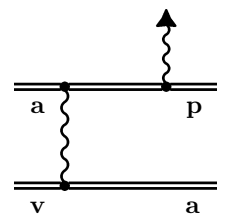

(5)

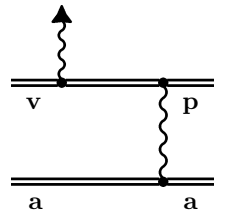

(2)

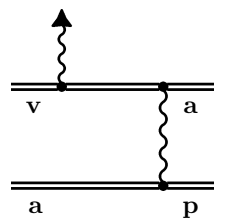

(6)

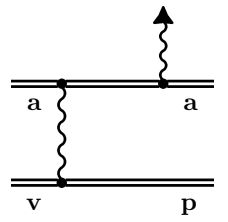

(3)

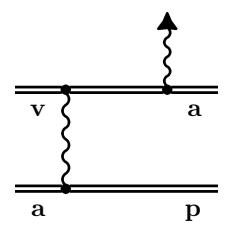

(7)

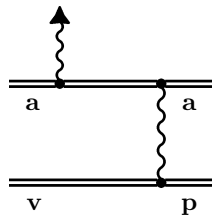

(4)

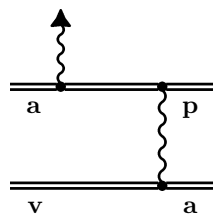

(8)

Figure 2: The one-photon exchange correction to the transition amplitude of the radiative recombination of an electron with a hydrogen-like ion. The double line indicates an electron propagating in the field of a nucleus. The wavy line with an arrow denotes the emitted photon. The incoming electron is denoted as $p, a$ is the initially bound (spectator) electron, and $v$ is the captured electron. From Ref. [एप].

Using the impulse approximation, the linear polarization of the REC photons in relativistic collisions of high $-Z$ projectiles with spin-polarized low- and medium- $Z$ target atoms has been investigated for a wide range of collision energies and impact parameters. From the results obtained in Refs. [ [1], [0], we concluded that the polarization parameters might be very sensitive to the impact parameter at which the ion-atom collision occurs and, hence, to a momentum distribution of particular atomic (target) orbitals. In contrast, if one does not have control over the collision impact parameter-as usually happens in modern experiments- the target effects appear to be of less importance. As such, our calculations suggest that differences between the REC linear polarization and that of the photons emitted in the course of the recombination of a free electron do not exceed $5-10 \%$ for light $-Z$ target atoms.

\subsection{Inter-electronic effects in projectile ions}

Apart from the analysis of the "target effects", an understanding of the many-body phenomena in the projectiles is also required for the application of the radiative electron capture as a probe process for measuring the ion spin polarization at storage rings. In order to explore these $e-e$ correlations, the rigorous quantum electrodynamic (QED) approach has been developed recently in Ref. [ㅁ] . As usual in such an approach, the interelectronic interactions are described by the exchange of the virtual photons. For heavy ions it is sufficient to consider the one-photon exchange only, since the exchange by two or more photons is suppressed by an additional factor of $1 / Z$. The set of Feynman diagrams representing these one-photon exchange corrections to the REC transition amplitude is depicted in Fig. ㅁ. By evaluating the corresponding matrix elements for the REC of ultimately helium-like ions, we were able to investigate how the tilt angle $\chi_{0}$ of the REC linear polarization is affected by the interelectronic effects. As seen from Fig. [1, where we displayed the relative difference between the predictions of the independent particle model, which neglects any 


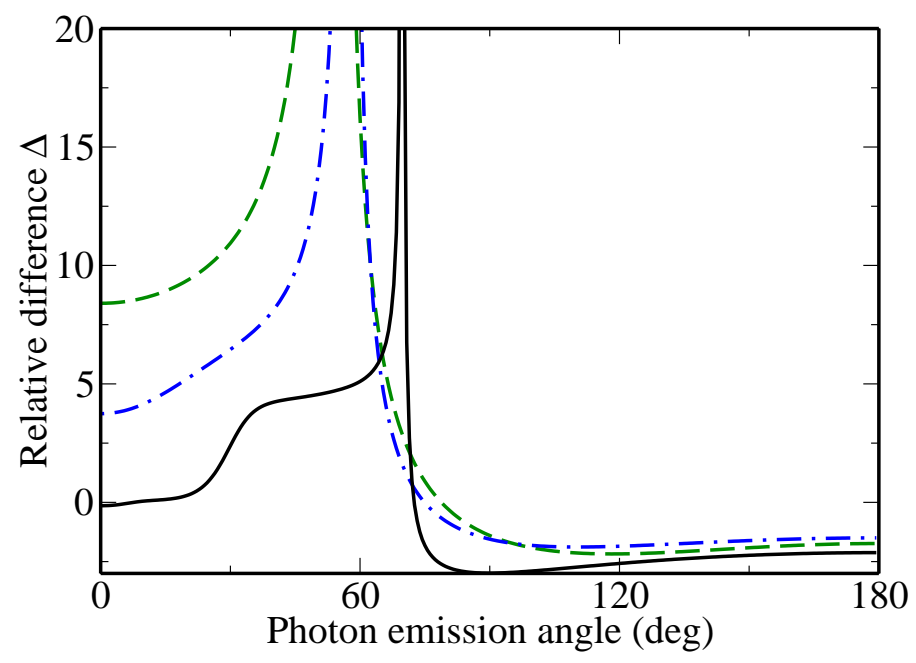

Figure 3: The relative difference $\Delta$ between the predictions of the independent particle model and the QED perturbative approach for the tilt angle $\chi_{0}$ of the REC linear polarization. Calculations are performed for the electron recombination into the ground state of completely polarized hydrogen-like bismuth ions with energies $T_{p}=100$ (dashed line), 300 (dash-and-dotted line) and $600 \mathrm{MeV} / \mathrm{u}$ (solid line). Results compiled from Ref. [एप]].

$e-e$ correlations, and the perturbative QED approach:

$$
\Delta=\frac{\chi_{0}^{(0)}-\chi_{0}}{\left|\chi_{0}^{(0)}\right|} \times 100 \%,
$$

the interelectronic interactions lead to the reduction of the tilt angle $\chi_{0}$. The most pronounced effects are observed for the forward photon emission, $5^{\circ} \lesssim \theta \lesssim 30^{\circ}$, where the difference between the predictions of the "zero-order" and QED theories may reach 15-20\% for mild collisions. One may note that $\Delta$ can enhance even further if the tilt angle $\chi_{0}$ becomes very small, $\left|\chi_{0}\right| \lesssim 0.5^{\circ}$ (see also left panel of Fig. W). This region, however, is not of interest for present-day experimental studies owing to the limited (angular) resolution of solid-state polarization detectors.

\section{Summary}

In conclusion, the application of the radiative electron capture for the spin-diagnostics of heavy ion beams at storage rings has been reviewed. Apart from the discussion of the general measurement scheme we questioned how the inter-electronic interactions affect the polarization transfer from the incident (polarized) ions to the emitted REC photons. These interactions occur both in a target atom and in an initially few-electron projectile ion. The analysis of the "target effects" has been successfully performed within the impact-parameter picture and impulse approximation, while a systematic QED treatment was carried out to understand the many-body phenomena in heavy ions. Taken together, these studies made it possible to provide accurate predictions for the polarization transfer in collisions of polarized hydrogen-like projectiles with low- $Z$ targets. The results obtained have opened up a unique possibility for high-precision beam diagnostics at storage 
ring facilities, which is a stringent requirement for exploring atomic PNC phenomena as well as searching for electric dipole moments of heavy nuclei.

\section{Acknowledgment}

The work reported in this paper was supported by the Helmholtz Gemeinschaft (Nachwuchsgruppe $\mathrm{VH}-\mathrm{NG}-421)$.

\section{References}

[1] L. N. Labzowsky, A. V. Nefiodov, G. Plunien, G. Soff, R. Marrus, and D. Liesen, Parity-violation effect in heliumlike gadolinium and europium, Phys. Rev. A 63 (2001) 054105

[2] A. V. Nefiodov, L. N. Labzowsky, D. Liesen, G. Plunien, and G. Soff, Nuclear anapole moments from beams of highly charged ions, Phys. Lett. B 534 (2002) 52

[3] L. Labzowsky and A. Prozorov, Parity nonconservation effects in the highly charged ions, J. Phys. Conf. Ser. 72 (2007) 012010

[4] A. Surzhykov, S. Fritzsche, Th. Stöhlker, and S. Tashenov, Application of radiative electron capture for the diagnostics of spin-polarized ion beams at storage rings, Phys. Rev. Lett. 94 (2005) 203202

[5] A. Prozorov, L. Labzowsky, D. Liesen, and F. Bosch, Schemes for radiative polarization of ion beams in storage rings, Phys. Lett. B 574 (2003) 180

[6] A. Bondarevskaya, A. Prozorov, L. Labzowsky, G. Plunien, D. Liesen, and F. Bosch, Theory of the polarization of highly charged ions in storage rings: Production, preservation, observation and application to the search for a violation of the fundamental symmetries, Phys. Rep. 507 (2011) 1

[7] A. Bondarevskaya, A. Prozorov, L. Labzowsky, G. Plunien, D. Liesen, and F. Bosch, Hyperfine quenching of polarized two-electron ions in an external magnetic field, Phys. Lett. A 372 (2008) 6642

[8] A. A. Bondarevskaya, L. N. Labzowsky, A. A. Prozorov, G. Plunien, D. Liesen, and F. Bosch, Linear polarization of $x$-ray photons in HFQ transitions of polarized He-like ions with application to the search for parity nonconservation effects, Phys. Scr. T144 (2011) 014007

[9] S. Tashenov et al., First measurement of the linear polarization of radiative electron capture transitions, Phys. Rev. Lett. 97 (2006) 223202

[10] U. Spillmann, H. Bräuning, S. Hess, H. Beyer, Th. Stöhlker, J.-Cl. Dousse, D. Protic, and T. Krings, Performance of a Ge-microstrip imaging detector and polarimeter, Rev. Sci. Instrum. 79 (2008) 083101

[11] A. Khaplanov, S. Tashenov, B. Cederwall, and G. Jaworski, A gamma-ray polarimeter based on a single segmented planar HPGe detector, Nucl. Inst. Meth. A 593 (2008) 459

[12] S. Tashenov, A. Khaplanov, B. Cederwall, and K.-U. Schässburger, Hard X-ray polarimetry by means of Rayleigh scattering, Nucl. Inst. Meth. A $\mathbf{6 0 0}$ (2009)

[13] G. Weber, H. Bräuning, S. Hess, R. Märtin, U. Spillmann, and Th. Stöhlker, Performance of a position sensitive $\mathrm{Si}(\mathrm{Li}) \mathrm{x}$-ray detector dedicated to Compton polarimetry of stored and trapped highly-charged ions, JINST 5 (2010) C07010

[14] G. Weber et al., Direct determination of the magnetic quadrupole contribution to the Lyman- $\alpha_{1}$ transition in a hydrogenlike ion, Phys. Rev. Lett. 105 (2010) 243002 
[15] S. Tashenov et al., Measurement of the correlation between electron spin and photon linear polarization in atomic-field bremsstrahlung, Phys. Rev. Lett. 107 (2011) 173201

[16] G. Weber et al., PEBSI - A Monte Carlo simulator for bremsstrahlung arising from electrons colliding with thin solid-state targets, Nucl. Instr. Meth. B in press

[17] J. Eichler and Th. Stöhlker, Radiative electron capture in relativistic ion-atom collisions and the photoelectric effect in hydrogen-like high-Z systems, Phys. Rep. 439 (2007) 1

[18] A. Surzhykov, S. Fritzsche, Th. Stöhlker, and S. Tashenov, Polarization studies on the radiative recombination of highly charged bare ions, Phys. Rev. A 68 (2003) 022710

[19] A. Surzhykov, A. N. Artemyev, and V. A. Yerokhin, Interelectronic interaction effects on the polarization of recombination photons, Phys. Rev. A 83 (2011) 062710

[20] A. N. Artemyev, A. Surzhykov, S. Fritzsche, B. Najjari, and A. B. Voitkiv, Target effects on the linear polarization of photons emitted in radiative electron capture by heavy ions, Phys. Rev. A 82 (2010) 022716 